\title{
Neuropsychological changes in a prospectively followed cohort of homosexual and bisexual men with and without HIV infection
}

\author{
Y. Stern, PhD; X. Liu, PhD; K. Marder, MD, MPH; G. Todak, CSW; \\ M. Sano, PhD; A. Ehrhardt, PhD; and J. Gorman, MD
}

\begin{abstract}
Article abstract-We evaluated neuropsychological test performance of 168 homosexual and bisexual men with and without human immunodeficiency virus (HIV) infection (113 HIV+ subjects and $55 \mathrm{HIV}-$ controls) over 4.5 years of semiannual follow-up. Analyses of the longitudinal data were performed by applying generalized estimating equations (GEEs) to regression analyses with repeated measures. Compared with the HIV - men, the HIV+ subjects performed more poorly on memory testing. Performance on all tests tended to improve over time, but this improvement was attenuated or eliminated in the HIV + group for tests of language and attention. Within the HIV+ subjects, improvement over time in tests of memory, executive function, language, and attention was attenuated or eliminated in patients with lower CD4 levels; more advanced HIV disease was associated with poorer memory and executive function and with attenuated or reduced learning effects for memory, motor speed, and language tests. Clinically significant neurologic findings were associated with worse memory and orientation and with attenuated or reversed learning effects for memory, language, and attention tests. There were 33 deaths in the HIV+ group. In the men who died, there was more rapid decline in executive, language, and attentional test performance. These observations remained significant after controlling for HIV disease severity. We conclude that HIV infecting the CNS results in progressive cognitive change that is closely associated with neurologic findings. In addition, our findings suggest a relation between more rapid cognitive progression and death.
\end{abstract}

NEUROLOGY 1995;45:467-472

We previously reported ${ }^{1}$ a cross-sectional analysis that demonstrated subtle differences between performance of homosexual men with and without human immunodeficiency virus (HIV) infection on a neuropsychological test battery. Test performance in the HIV + subjects correlated significantly with an index of neurologic signs and a measure of cognitive complaints. We have now followed this cohort of homosexual and bisexual men for up to 4.5 years and report data regarding change in performance over time as a function of HIV status, disease severity, and neurological signs and symptoms.

We have also reported that defective performance on neuropsychological tests at the subject's initial visit increased the relative risk of death over 3 years, ${ }^{2}$ which implies that HIV in the CNS is associated with more severe disease. We report anal- yses here that investigate the relative rapidity of cognitive decline in HIV+ members of our cohort who did, and did not, die.

Methods. Subjects. The recruitment and baseline characteristics of this cohort have been previously described. ${ }^{3}$ Two hundred seven homosexual and bisexual men were recruited in late 1987 and early 1988 to participate in a 5-year follow-up study of HIV-infected subjects $(\mathbf{n}=123$ ) and HIV - controls $(n=84)$. The majority of subjects were recruited by word of mouth; two announcements were placed in gay-oriented monthly newsletters and one in a newspaper. All subjects were volunteers and gave informed consent. Subjects were paid for their participation in the study. One hundred sixty-eight subjects were entered into the longitudinal phase of the study (113 HIV + and $55 \mathrm{HIV}-$ ) and were examined every 6 months for up to 10 visits.

Assessments. At each visit, subjects received a stan-

From the Department of Neurology (Drs. Stern, Marder, and Sano), the Gertrude F. Sergievsky Center (Drs. Stern, Marder, and Sano), the HIV Center for Clinical and Behavioral Studies (Drs. Stern, Liu, Marder, Sano, Ehrhardt, and Gorman, and G. Todak), and the Department of Psychiatry (Drs. Storn, Ehrhardt, and Gorman), College of Physicians and Surgeons of Columbia University, New York, NY.

Supported by NIMH/NIDA P50-MH43520 to the HIV Center for Clinical and Behavioral Studies, NIMH T32-MH19139-05, NIMH 3-M01-RR00645, and NIA AG10963.

Reccived June 16, 1994. Accepted in final form Septomber 10, 1994.

Address correspondence and reprint requests to Dr, Yaakov Stern, Gertrude H. Sergievsky Center, 630 W. 168 th Street, New York, NY 10032. 
dardized medical examination for the purpose of staging, and also received neurologic, neuropsychological, psychiatric, and psychosexual assessments. These assessments have been described previously ${ }^{1,3}$; relevant assessments are summarized below.

Medical assessment. Physicians performed physical examinations targeted at HIV-related symptoms and signs. Subjects who met 1987 Centers for Disease Control criteria for acquired immunodeficiency syndrome (AIDS) ${ }^{4}$ at baseline were not included in the study, with two exceptions. Because of the difficulty in distinguishing clinically between oral and esophageal candidiasis on routine physical examination, we did not exclude men with esophageal candidiasis, which is, on its own, a criterion for AIDS. We also did not use the presence of cognitive impairment as an exclusion criterion because the purpose of the study was to assess nervous system abnormalities in relatively medically asymptomatic individuals. Blood for CD4 counts was obtained at each visit.

Following each study visit, HIV+ patients were classified by disease stages based on previously described criteria. ${ }^{5}$ For the present analyses we considered three severity stages: stage 1 , asymptomatic or mildly symptomatic; stage 2, signs and symptoms consistent with AIDS-related complex (ARC); and stage 3, AIDS.

Neurologic assessment. All neurologic examinations were performed by neurologists who were blind to the HIV status of the subjects. In the interests of maintaining blinding, the neurologic examinations were performed prior to the elicitation of symptoms. Each neurologic sign was coded separately so that individual signs could be examined and factor scores could be created based on individual items. A standard neurologic examination was performed. Items from the motor portion of the Unified Parkinson's Disease Rating Scale ${ }^{6}$ were included to assess extrapyramidal function. An overall measure of neurologic disability was calculated for each subject, based on the Kurtzke Expanded Disability Status Scale for multiple sclerosis. ${ }^{7}$ The Kurtzke scale reflects both the number of areas of neurologic dysfunction (ie, pyramidal tract, cerebellum, brainstem, etc) and the severity of impairment. Derivation of the Kurtzke scale for use in HIV infection has been previously described. ${ }^{1}$ We subdivided subjects into those with and without clinically significant neurologic findings using a score of 3 or above on the Kurtzke scale as the cutpoint.

Neuropsychological assessment. The neuropsychological battery was also administered with the testers blind to HIV status. Although a larger battery was administered at the initial visit, ${ }^{1}$ the following tests were consistently included in the battery that was administered at all visits:

The 10 orientation items from the Mini-Mental State Examination ${ }^{8}$ were used to assess orientation to time and place. Attention was assessed with two types of cancellation tasks, one utilizing a shape and another a letter triad as targets, ${ }^{9}$ and with the Wechsler Adult Intelligence Scale-Revised Digit Symbol subtest. ${ }^{10}$ Verbal fluency was assessed with the Controlled Oral Word Association test $(60$ seconds for each of the letters $C, F$, and $L)^{11}$ and animal naming. ${ }^{12}$ The Odd Man Out Test ${ }^{13}$ was used to assess executive or "frontal lobe" function. In this test of the ability to switch set, the subject is asked to utilize a consistent selection rule to determine which of three symbols on a card does not belong with the other two. The subject must then systematically alternate between two selection rules over four trials, each consisting of 10 cards. The Trail Making Test was also adminis- tered as a test of speeded performance and, in part B, of rapid sequencing and set-shifting. ${ }^{14}$ Motor speed and praxis were assessed with the Purdue Pegboard. ${ }^{15}$ This test was administered only in the first seven visits. A 12item, six-trial selective reminding test assessed verbal memory. ${ }^{16}$ Fifteen minutes later, the subjects' delayed recall for the word list was assessed and words not recalled were administered in multiple-choice arrays to assess delayed recognition.

To reduce the number of test scores in our analyses, we performed a factor analysis of the test scores at the baseline visit and assessed stability of the factors at the follow-up visits. Using these factor analyses as a guide, six neuropsychological factor scores were constructed. These included (1) memory-selective reminding test total score; (2) motor speed-Purdue Pegboard, pegs with dominant hand; (3) executive-Odd Man Out total score and Trails B time; (4) language-number of words reported on animal naming and Controlled Oral Word Association; (5) attention-Digit Symbol age-scaled score, shape and letter cancellation time; and (6) orientationorientation score. To calculate factor scores for each patient, each component score was first transformed into a $\mathrm{z}$ score based on the mean and standard deviation of test scores of all subjects assessed at the baseline visit. The $z$ scores were adjusted for direction (ie, whether higher scores indicate better or worse performance). Factor scores then were calculated as sums of the $\mathrm{z}$ scores for measures within each factor. The range of scores on the orientation test was severely restricted (ie, few errors were made). For statistical purposes, performance on this factor was expressed as the number of errors. In this case only, higher scores represented poorer performance.

Statistical analysis. Analyses of the longitudinal data were performed by applying generalized estimating equations (GEEs) to regression analyses with repeated measures. ${ }^{17}$ This statistical method takes into account the multiple visits per subject and the fact that the characteristics of a single individual over time are likely to be correlated with one another. The repeated measures for each subject (up to 10 per variable) are treated as a cluster. A second advantage of GEEs is that this method takes into account the status or changing value of each covariate at each visit. Tabled values for regression analyses involving the factor scores are regression coefficients and their standard error. All analyses were controlled for age and education.

The distributions of all factors except for orientation were approximated by a normal distribution. As noted above, scores for the orientation factor had an attenuated range and were converted to error scores; their distribution was approximated by a Poisson distribution.

In the initial set of analyses, we examined test factor performance over time as a function of serostatus. The regression models provided estimates of the association of neuropsychological factor scores with serostatus, follow-up time, and the interaction of serostatus and time. A significant interaction of HIV status and time would indicate differential rates of change in a factor score as a function of HIV status.

The remaining analyses were restricted to the $\mathrm{HIV+}$ subjects. Two sets of regression analyses explored the contribution of disease severity (CD4 count $\leq 200$ cells $/ \mathrm{mm}^{3}$ versus $>200$ cells $/ \mathrm{mm}^{3}$, or disease stage) by including time, the disease severity measure, and the interaction of time and severity in the model. A third set of analyses evaluated the relationship between neuropsychological performance and clinically significant neuro- 
Table 1. Baseline characteristics for HIV- and HIV+ subjects followed longitudinally

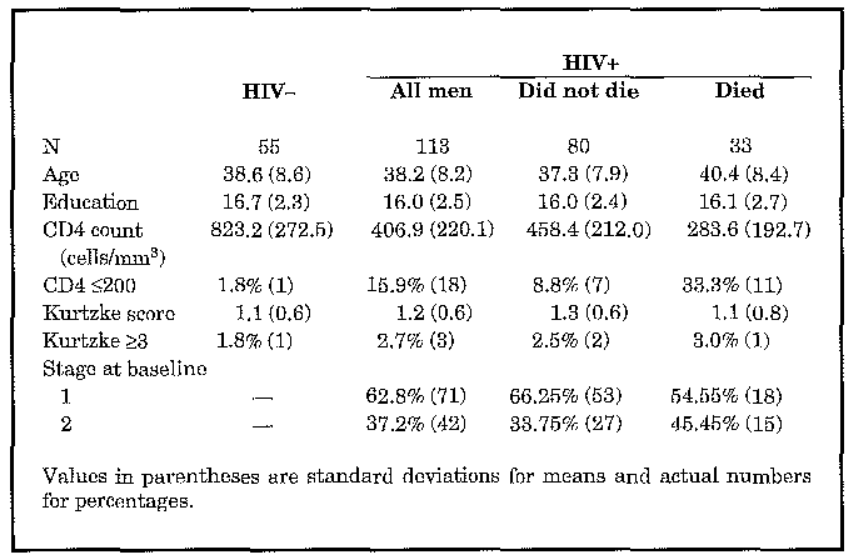

logic findings (Kurtzke score $<3$ versus $\geq 3$ ) by including time, neurologic impairment, and their interaction in the model.

A final set of regression models was constructed to evaluate possible differences in test performance or progression in subjects who did and did not die during the study period. Similar to the analyses described above, the models initially explored the main effects of time, death, and their interaction. Subsequent analyses controlled for disease stage or CD4 and Kurtzke scores in order to evaluate the relationship between neuropsychological performance and death independent of disease severity.

Results. Demographics. One hundred sixty-eight subjects (113 HIV+ and $55 \mathrm{HIV}-$ ) were included in the longitudinal follow-up cohort. The baseline characteristics of these HIV+ and HIV - subjects are presented in table 1. Although 84 HIV- men were assessed at the baseline visit, the study design called for follow-up of only 55. There was no significant difference between the HIV - men who were and were not followed on any demographic or neuropsychological measure.

One of the HIV - and 33 of the HIV+ subjects died over 5 years. Three of the HIV+ subjects died after completing their 10th visit. Baseline CD4 counts of subjects who died differed significantly from those of HIV - subjects as well as from the other HIV+ subjects (table 1). Other baseline characteristics did not differ between those who did and did not die.

In addition to subject loss due to death, some subjects were lost to follow-up or refused to continue as the study progressed, and some tests could not be completed at some visits. The largest attrition was toward the end of the follow-up period; by visit 9, data were available for $40 \mathrm{HIV}-$ and 62 HIV+ subjects, and by visit 10 , data were available for $28 \mathrm{HIV}$ - subjects and $51 \mathrm{HIV}+$ subjects.

Contrasts of HIV-and HIV+ subjects. Across all visits, the HIV+ subjects performed more poorly than the HIV - subjects on the memory factor (table 2). In both HIV - and HIV+ subjects, performance scores for all factors except orientation improved over time. There was a significant serosta-
Table 2. Regression coefficients (standard error) assessing the association of each factor score with serostatus, follow-up time, and their interaction*

\begin{tabular}{|lccc|}
\hline Factor & Serostatus & Time & $\begin{array}{c}\text { Serostatus } \\
\times \text { time }\end{array}$ \\
Memory & $-0.40(0.13) \dagger$ & $0.13(0.03) \dagger$ & $-0.03(0.03)$ \\
Motor speed & $-0.15(0.15)$ & $0.29(0.05) \dagger$ & $0.01(0.06)$ \\
Executive & $-0.10(0.24)$ & $0.28(0.03) \dagger$ & $-0.06(0.04)$ \\
Language & $-0.24(0.27)$ & $0.28(0.03) \dagger$ & $-0.09(0.04) \dagger$ \\
Attention & $0.39(0.38)$ & $0.39(0.05) \dagger$ & $-0.24(0.08) \dagger$ \\
Orientation & $-0.51(0.37)$ & $-0.04(0.10)$ & $0.24(0.13)$ \\
& & \\
* All analyses control for ago and education. & \\
$\uparrow p<0.05$. & & & \\
\end{tabular}

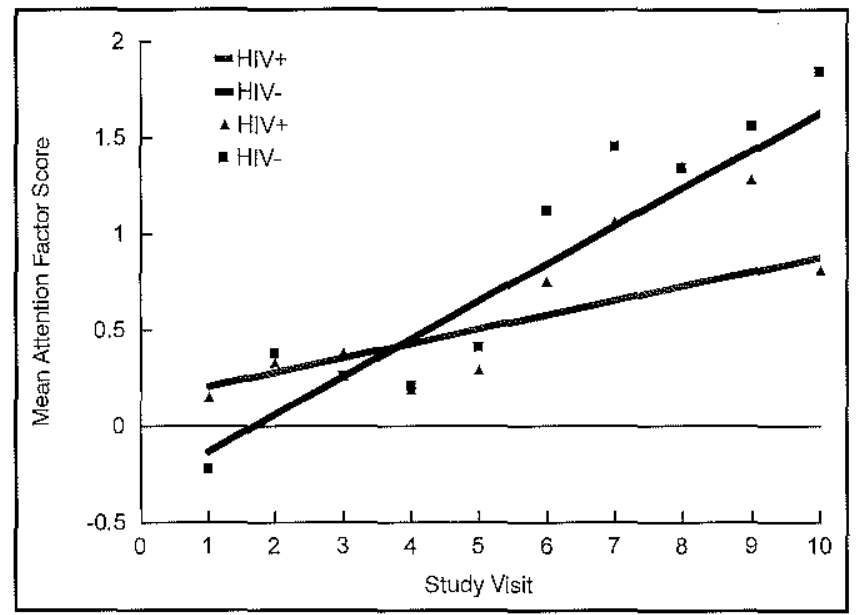

Figure. Mean attention factor scores for HIV- and HIV+ subjects at each study visit. Regression lines are derived from the generalized estimating equations model.

tus $\times$ time interaction for the language and attention factors. The negative interaction indicates that for these factors there was less improvement in performance over time in the HIV+ subjects. The figure demonstrates this interaction for the attention factor scores.

Analyses within HIV+ subjects. We next evaluated the contribution of disease severity, measured by CD4 levels stratified at $\leq 200$ cells $/ \mathrm{mm}^{3}$ and $>200$ cells $/ \mathrm{mm}^{3}$. For these and all subsequent analyses, only the regression coefficients for the interaction with follow-up time are tabulated. Performance on all factors but orientation improved over time. There was a significant CD4 level $\times$ time interaction for the memory, executive, language, and attention factors, indicating an attenuated learning effect or more rapid decline in performance over time when the CD 4 count was below 200 cells $/ \mathrm{mm}^{3}$ (table 3). Since there was not a significant time effect for orientation, only the CD4 stratification was retained in the model, and no significant effect was noted.

A similar set of models was constructed that measured severity by disease stage. In this model, 
Table 3. In HIV+ subjects only, regression coefficients (standard error) for the interaction of follow-up time with selected clinical variables*

\begin{tabular}{|c|c|c|c|c|c|}
\hline Factor & CD4 $\times$ Time & Stage $2 \times$ Time & Stage $3 \times$ Time & Kurtzke score $\times$ Time & Died $\times$ Time \\
\hline Memory & $-0.16(0.05) \dagger$ & $-0.13(0.04) \dagger$ & $-0.41(0.11) \dagger$ & $-0.36(0.14) \dagger$ & $0.03(0.07)$ \\
\hline Motor speed & $-0.10(0.08)$ & $0.05(0.08)$ & $-0.37(0.12) \dagger$ & $-0.20(0.14)$ & $-0.11(0.10)$ \\
\hline Executive & $-0.19(0.08) \uparrow$ & $-0.01(0.06)$ & $0.22(0.14)$ & $-0.18(0.22)$ & $-0.19(0.08) \dagger$ \\
\hline Language & $-0.16(0.06) \dagger$ & $-0.11(0.06)$ & $-0.30(0.12) \dagger$ & $-0.43(0.15) \dagger$ & $-0.25(0.07) \dagger$ \\
\hline Attention & $-0.40(0.17) t$ & $-0.03(0.11)$ & $-0.15(0.21)$ & $-1.21(0.49)+$ & $-0.62(0.18)+$ \\
\hline \multicolumn{6}{|c|}{$\begin{array}{l}\text { : Variables include CD4 count (stratified at } 200 \text { cells/mmis), disease stage, the presence or absence of clinically signifieant neurologic findings } \\
\text { (Kurtzke score stratified at } 3 \text { ), and whether or not the subject died. The interaction coefficients are from separate regression models assessing } \\
\text { the association of each factor: score with follow-up time, the clinical measure, and the interaction of time and the clinical measure. All analyses } \\
\text { control for age and education. }\end{array}$} \\
\hline
\end{tabular}

performance on all factors but orientation improved significantly over time, and the time variable was excluded from the model assessing orientation. In comparison with stage 1 (asymptomatic or mildly symptomatic), stage 2 (ARC) was associated with more orientation errors, and stage 3 (AIDS) was associated with poorer performance on memory and executive function. There was a significant stage $2 \times$ time interaction for memory, indicating an attenuated learning effect in patients with ARC, and a significant stage $3 \times$ time interaction for memory, motor speed, and language, indicating an attenuated learning effect or decline in performance for patients with AIDS (table 3). We evaluated the possibility that the relationship between disease severity and motor speed might be due to increased numbers of men with peripheral neuropathy. Peripheral neuropathy was rare (seven men over all visits), and its presence was not related to motor speed.

In order to investigate the relationship between the presence of neurologic signs and cognitive performance, we constructed a model that included the Kurtzke scale, dichotomized at a score of 3 and above to represent the presence or absence of clinically significant neurologic problems. Overall, all factors but orientation improved over time, and the time variable was excluded from the model assessing orientation. The presence of significant neurologic signs was associated with poorer performance on the memory factor and more orientation errors. There was a significant Kurtzke score $\times$ time interaction for the memory, language, and attention factors, indicating that the learning effect for these factors was attenuated or eliminated in subjects who had or developed clinically significant neurologic signs (table 3 ). To evaluate the possibility that the relationship between neurologic signs and neuropsychological factors was actually due to neurologic signs being more common in advanced disease, we incorporated disease stage into the regression model. The Kurtzke score $\times$ time interactions remained significant.

Factor scores in HIV+ subjects who did and did not die. There were 33 deaths in the HIV+ group. Average time between final testing and death was approximately 8 months. Table 1 presents demographic data at baseline for HIV+ subjects who did and did not die. There was a significant death $\times$ time interaction for the executive, language, and attention factors, indicating that the improvement in factor scores generally seen over time was attenuated in men who died (table 3). A subsequent analysis controlled for disease severity and established that for any level of disease severity, there was poorer performance and more rapid progression of neuropsychological problems in men who died.

Discussion. The present findings confirm and extend previous observations of cognitive change in HIV infection among well-educated, high-socioeconomic-status homosexual and bisexual men. None had AIDS at the beginning of the study, and 43 were medically asymptomatic. If neuropsychological test performance is considered to be an indicator of the effects of HIV on the CNS, the initial results would be expected to be quite subtle. Still, we reported $^{1}$ small but statistically significant differences between the performance of HIV - and HIV+ subjects on some tests at baseline. These included performance differences on tests of memory, language, attention, abstract reasoning, speeded processing, and executive function. Because tests were administered every 6 months, subjects would be expected to have become quite familiar with the test materials. In fact, a striking feature of the present prospective analyses is the general trend for improved performance over time on all the neuropsychological test factors considered. The potential effects of HIV infection on performance therefore must be judged against a background of improving scores. In our comparison of the performance of the HIV - and HIV+ groups over time, we found that memory was poorer in the HIV+ groups at all visits. In addition, for the language and attention factors, the trend toward improved performance over time was significantly attenuated in the HIV+ subjects. We hypothesize that these cognitive effects are an early sign of a more general effect of HIV on the CNS. The generally poorer performance of the $\mathrm{HIV}+$ subjects on memory tests has been noted by 
several groups in cross-sectional studies, ${ }^{1,18}$ The differential change in language and attention also parallels our previous findings.

We used factor scores to summarize neuropsychological test data. While the name of each factor reflects our view of the primary cognitive function that is tapped, it is certainly the case that these factors reflect multiple processes. For example, the tests included in the attention factor also require psychomotor speed and scanning.

To better understand HIV's effects on test performance, subsequent analyses focused on the HIV + subjects only. The significant interactions between time and disease severity (expressed either in terms of disease stage or CD4 counts) for many neuropsychological test factors indicated that the development of more severe disease was associated with more marked attenuation of the learning effect and, in most cases, with decline in performance over time. This observation parallels that made by many other investigators ${ }^{19-22}$ primarily using crosssectional data: the effect of HIV on neuropsychological performance is more marked in the later stages of the disease. More important, the present observations confirm in prospectively collected data that progression in HIV disease severity is associated with more rapid progression of neuropsychological changes.

To further test the idea that HIV can have a specific effect on the CNS, we investigated the relation between the emergence of neurologic abnormalities and neuropsychological change. We found that patients who had or developed neurologic abnormalities were more likely to perform more poorly on the neuropsychological battery. Further, this relationship remained after controlling for disease severity, suggesting that at any stage of severity there was an association between neurologic and neuropsychological abnormalities. This observation is in agreement with our cross-sectional analyses of the baseline data, which suggested that HIV's effect on the CNS will be manifested by both cognitive and neurologic changes. We designed the present analyses to evaluate the association of the neurologic and neuropsychological manifestations of HIV infection at any visit, but cannot address their relative timing. The latter issue is particularly complex in the earlier stages of the disease because neurologic signs are not always present consistently from one visit to the next.

We also investigated the relationship between mortality and change in cognitive function over time. We previously reported that defective performance on neuropsychological testing or symptomatic motor complaints at the initial visit were associated with increased relative risk of death, even after controlling for disease severity indices. The present analyses demonstrate that the subjects who died had an attenuated learning effect for executive tasks and relative declines in performance on language and attention tasks. These observations remained significant after controlling for disease severity. These findings are unlikely to represent marked changes that might occur in test performance during severe illness immediately prior to death because the average time between the final study visit and death was approximately 8 months. Because we studied only ambulatory subjects and did not offer treatment, subjects often terminated participation when they became more ill or had difficulty traveling to the medical center, and we did not have an opportunity to test severely ill subjects. Therefore, our findings suggest that when HIV affects the CNS (as indicated by neuropsychological and neurologic changes), it is associated with more rapid disease progression and death.

The differences detected between HIV+ and HIV - men may be underestimated because HIV+ subjects were often not examined when they became extremely ill. This may also explain, in part, the observed general improvement in test performance over time: there may have been differential loss to follow-up of the most cognitively impaired individuals. Therefore, the neuropsychological changes observed in this study are relatively subtle and may underestimate actual changes to some degree. An alternate possibility is that men with systemic disease might not have returned for followup while those with cognitive changes did. We consider this unlikely, particularly since our analyses indicate a relationship between disease severity and cognitive change. In any case, the present findings confirm our initial cross-sectional observations that HIV in the CNS results in progressive cognitive changes that are closely associated with neurologic findings.

\section{References}

1. Stern Y, Marder K, Bell K, et al. Multidisciplinary baseline assessment of homosexual men with and without human immunodeficiency virus infection. III. Neurologic and neuropsychologic findings. Arch Gen Psychiatry 1991;48:131-138.

2. Mayeux R, Stern $Y$, Tang MX, et al. Mortality risks in gay men with human immunodeficiency virus infection and cognitive impairment. Neurology 1993;43:176-182.

3. Gorman JM, Kertzner R, Todak G, et al. Multidisciplinary baseline assessment of homosexual men with and without human immunodeficiency virus infection. I. Overview of study design. Arch Gen Psychiatry 1991;48:120-123.

4. Centers for Disease Control. CDC classification system for human T-lymphotropic virus type III/ymphadenopathy-associated virus infections. MMWR 1986;35:334-339.

5. Gorman JM, Lange M, Dobkin J, et al. Development and characteristics of a medical staging system for HIV infection. Int J Methods Psychiatr Res 1992;2:117-124.

6. Fahn S, Marsden C, Calne D, eds. Recent developments in Parkinson's disease. Florham Park, NJ: Macmillan Healthcare Information, 1987;2:153-163.

7. Kurtzlke JF. Rating neurologic impairment in multiple sclerosis: an expanded disability status scale (EDSS). Neurology 1983;33:1444-1452.

8. Folstein MF, Folstein SE, McHugh PR. 'Mini-mental state.' A practical method for grading the cognitive state of patients for the clinician. J Psychiatr Res 1975;12:189-198.

9. Sano M, Rosen W, Mayeux R. Attention deficits in Alzheimer's disease. Presented at the 92nd annual meeting of the American Psychological Association, Toronto, ON, 
Canada, 1984.

10. Wechsler D. Wechsler aduIt intelligence scale-revised. New York: Psychological Corporation, 1981.

11. Benton A. FAS test. In: Spreen O, Benton A, eds. Neurosensory center comprehensive examination for aphasia. Victoria, BC, Canada: University of Victoria, 1967.

12. Goodglass H, Kaplan D. The assessment of aphasia and re lated disorders. 2nd ed. Philadelphia: Lea and Febiger, 1983.

13. Flowers K, Robertson C. The effect of Parkinson's disease on the ability to maintain a mental set. J Neurol Neurosurg Psychiatry 1985:48:517-529.

14. Reitan RM, Wolfson D. The Halstead-Reitan Neuropsychological Test Battery: theory and clinical interpretation. Tucson, AZ: Neuropsychology Press, 1985.

15. Tiffin J. Purdue Pegboard: examiner manual. Chicago, IL: Science Research Associates, 1968.

16. Buschke H, Fuld PA. Evaluating storage, retention, and retrieval in disordered memory and learning. Neurology $1974 ; 24: 1019-1025$.

17. Liang KY, Zeger SL. Longitudinal data analysis using gen- eralized linear models. Biometrika 1986;73:13-22.

18. Wilke FL, Eisdorfer CE, Morgan R, Lowenstein DA, Szapocznik J. Cognition in early human immunodeficiency virus infection. Arch Neurol 1990;47:433-440.

19. McArthur JC, Cohen BA, Selnes OA, et al. Low prevalence of neurological and neuropsychological abnormalities in otherwise healthy HIV-1 infected individuals: results from the Multicenter AIDS Cohort Study. Ann Neurol 1988;45:954958.

20. Goethe KE, Mitchell JF, Marshall DW, et al. Neuropsychological and neurological function of human immunodeficiency virus seropositive individuals. Arch Neurol 1989;46:129-133.

21, Grant 1, Atkinson J, Hesselink J, et al. Evidence for early central nervous system involvement in the acquired immunodeficiency syndrome and other HIV infections. Ann Intern Med 1987;107:828-836.

22. Janssen RS, Saykin AJ, Cannon L, et al. Neurological and neuropsychological manifestations of HIV-1 infection: association with AIDS-related complex but not asymptomatic HIV-1 infection. Ann Neurol 1989;26:592-600.

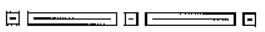

CReprinted from NEUROLOGY, Volume 45, Number 3, March 1995 aN ADVANSTAR PUBLICATION Printed in U.S.A. 